\title{
Nonlinear excitations in $\mathrm{CsNiF}_{3}$ in magnetic fields perpendicular to the easy plane
}

\author{
M. Orendáč, ${ }^{1}$ A. Orendáčová, ${ }^{1}$ E. Čižmár, ${ }^{2}$ J.-H. Park, ${ }^{2}$ A. Feher, ${ }^{1}$ S. J. Gamble, ${ }^{2}$ S. Gabáni,${ }^{3}$ K. Flachbart ${ }^{3}$ \\ J. Karadamoglou, ${ }^{4}$ M. Poirier, ${ }^{5}$ and M. W. Meisel $^{2}$ \\ ${ }^{1}$ Faculty of Science, P. J. Šafárik University, Park Angelinum 9, 04154 Košice, Slovakia \\ ${ }^{2}$ Department of Physics and Center for Condensed Matter Sciences, University of Florida, Gainesville, FL 32611-8440, USA \\ ${ }^{3}$ Institute of Experimental Physics, Slovak Academy of Sciences, Watsonova 47, 04353 Košice, Slovakia \\ ${ }^{4}$ Institut Romand de Recherche Numérique en Physique des Matériaux (IRRMA), EPFL, 1015 Lausanne, Switzerland \\ ${ }^{5}$ Centre de Recherche en Physique du Solide, Département de Physique, Univerité de Sherbrooke, J1K 2R1 Québec, Canada
}

(November 13, 2018)

\begin{abstract}
Experimental and numerical studies of the magnetic field dependence of the specific heat and magnetization of single crystals of $\mathrm{CsNiF}_{3}$ have been performed at $2.4 \mathrm{~K}, 2.9 \mathrm{~K}$, and $4.2 \mathrm{~K}$ in magnetic fields up to $9 \mathrm{~T}$ oriented perpendicular to the easy plane. The experimental results confirm the presence of the theoretically predicted double peak structure in the specific heat arising from the formation of nonlinear spin modes. The demagnetizing effects are found to be negligible, and the overall agreement between the data and numerical predictions is better than reported for the case when the magnetic field was oriented in the easy plane. Demagnetizing effects might play a role in generating the difference observed between theory and experiment in previous work analyzing the excess specific heat using the sine-Gordon model.
\end{abstract}

75.40Cx, 75.40.Mg, 75.30.Et, 75.10.Pq

\section{INTRODUCTION}

Significant developments in solid state physics have occurred when nonlinear effects have been taken into consideration. Low dimensional magnets have proven to be excellent model systems for these studies since long-range ordering is suppressed, whereas short-range ordering, if sufficiently pronounced, gives rise to nonlinear behavior. In this respect, spin chains garner special importance, and the interest in these one-dimensional (1D) magnets was piqued by Mikeska's prediction of the contribution of thermally excited solitons to the dynamics of a planar spin chain. ${ }^{1}$ These excitations are expected to appear in a symmetry breaking magnetic field oriented in the easy plane of the chain. Since the compound $\mathrm{CsNiF}_{3}$ is a good realization of an $S=1$ Heisenberg planar ferromagnetic chain, it has become one of the most intensively studied quasi-1D systems. Its magnetic properties in a magnetic field oriented in the easy plane have been investigated by different theoretical approaches ${ }^{2,3}$ and various experimental techniques. More specifically, the presence of sine-Gordon solitons was assumed in the interpretation of the central peak in the energy spectrum of slow neutrons ${ }^{4}$ of the temperature and field dependences of the spin-lattice relaxation time studied by NMR, ${ }^{5}$ and of the excess magnetic specific heat. ${ }^{6}$ In addition, the suppresion of the energy gap in the excitation spectrum of $\mathrm{CsNiF}_{3}$ in a magnetic field oriented in the easy plane was analyzed using quantum sine-Gordon field theory. ${ }^{7}$

Although the sine-Gordon model has been widely applied in the analysis of the data obtained on several Heisenberg chain systems, the existence of sine-Gordon solitons has not been proven unambiguously. For example, the temperature and magnetic field dependences of the intensity in the central peak observed by neutron scattering in $\mathrm{CsNiF}_{3}$ is better described by the spin-wave prediction in comparison with a model assuming scattering from a dilute gas of solitons. ${ }^{8}$ Furthermore, reasonable quantitative agreement between the experimentally measured peak of the excess specific heat and the corresponding theoretical prediction could be obtained only after an artificial renormalization of the soliton energy, ${ }^{6}$ and this aspect has been the subject of considerable debate. ${ }^{9,10}$ It is noteworthy that a similar situation was found for the $S=5 / 2$ Heisenberg planar ferromagnet TMMC. ${ }^{11}$ In other work, optical studies were initially interpreted in terms of magnon-soliton scattering, ${ }^{12}$ but were subsequently shown to be explainable by multimagnon process. ${ }^{13}$ Recent theoretical progress in calculating the magnon dispersion and thermodynamic properties of $\mathrm{CsNiF}_{3}$ using a transfer matrix renormalization group algorithm ${ }^{3}$ has allowed for the comparison of the experimental data with the exact theoretical predictions. Nevertheless, using generally accepted values of the exchange coupling $J$, the single-ion anisotropy $D$, and the $g$ factor for $\mathrm{CsNiF}_{3}$, only qualitative agreement was found for the magnetic field dependence of the specific heat.

Since solitons are predicted to appear when the magnetic field is oriented in the easy plane, less attention has been devoted to the situation when the field is oriented perpendicular to the easy plane. Apart from the experimental study of the temperature dependence of the susceptibility ${ }^{14}$ and the field dependence of the magnetization ${ }^{15}$ for such a field orientation, other work focused predominantly on mapping the magnetic phase diagram obtained from the anomalous behavior of the elastic constants. ${ }^{16}$ In addition, a theoretical treatment addressed the field induced instability of the threedimensional (3D) antiferromagnetically ordered phase at $T<T_{N}=2.7 \mathrm{~K}^{17}$ 
In fact, the situation is significantly richer than initially expected due to the formation of nonlinear spin modes when the magnetic field is orientated perpendicularly to the easy plane. ${ }^{3}$ More specifically, nonlinear effects lead to the formation of a characteristic double peak in the magnetic field dependence of the specific heat in the vicinity of a critical field $B_{c}$ at which the ground state is changed. The existence of the double peak has already been predicted in $S=1 / 2 \mathrm{XY}$ chains as well as $S=1 / 2$ Heisenberg chains with Ising anisotropy. ${ }^{18,19}$ The proposed behavior of the specific heat significantly differs from that obtained in the dilute-magnon approximation, where a single broad maximum at $B_{c}$ is predicted. The motivation of our experimental study of $\mathrm{CsNiF}_{3}$ was to search for the existence of the predicted double peak structure in the field dependence of the specific heat and to examine the thermodynamic response at several temperatures near $T_{N}$. As we will present in the following sections, our results confirm the existence of the theoretically predicted double peak structure. Moreover, the numerical analysis of our results indicates that the previously reported discrepancies between the excess specific heat data and the corresponding theoretical predictions may be partially attributed to demagnetization effects.

\section{EXPERIMENTAL DETAILS}

The single crystals of $\mathrm{CsNiF}_{3}$ used for our work were light green and transparent throughout their volume, albeit with some internal cleavage planes noticeable. Due to the highly hydroscopic nature of the material, all samples were kept in paraffin oil until they were ready to be mounted. For the specific heat study, a single crystal of mass $8.44 \mathrm{mg}$ and approximate dimensions $7 \times 0.5 \times 0.5 \mathrm{~mm}^{3}$ was used. This specimen was attached to a simple homemade microcalorimeter using Epotek E4110 silver epoxy. The calorimeter consisted of a sapphire disk that supported a heater and a thermometer, both made from $\mathrm{RuO}_{2}$ chip resistors. The thermometer was calibrated against a commercial Cernox thermometer (Lakeshore CX-1030CD) which was located near the calorimeter. The position of the calorimeter was fixed by a holder made of Vespel SP-1 that was needed to prevent motion in the magnetic field. The holder and the manganin wires from the thermometer and heater served as the thermal link to the stable reservoir. At $4.2 \mathrm{~K}$, the thermal conductivity of the manganin wires $(0.15 \mu \mathrm{W} / \mathrm{K})$ represented only about $5 \%$ of that of the vespel holder $(3 \mu \mathrm{W} / \mathrm{K})$. Consequently, the resultant value of the total thermal conductivity can be considered to be field independent. The resistance of the thermometer was measured using a Wheastone bridge, with a PAR 124A lockin amplifier serving as a null detector. While monitoring the cooling curve of a measurement, the analog output of the lock-in was read by a HP 3457A multimeter. The same device was used for measuring the voltage on the heater during the heating step, and the current was provided by a Keithley Model 220 current source. Consequently, the temperature and magnetic field dependences of the heater resistance did not influence the accuracy of the measurements. In the measuring cycle, the relative change of the temperature of the sample ranged from $3 \%$ to $5 \%$. The specific heat was calculated from the ratio of the characteristic cooling relaxation time and the thermal conductivity of the link. We estimate the overall accuracy of the measurement to be better than $5 \%$.

For the estimation of the correction due to demagnetizing effects, the field dependence of the magnetization was studied up to $7 \mathrm{~T}$ at several selected temperatures using a Quantum Design SQUID magnetometer. For these measurements, a single crystal, with dimensions similar to those used for specific heat studies, was placed in a polyethylene vial that was held by a straw. The background contribution from the vial and straw was independently measured to be less than $1 \%$ of the total signal and was subtracted.

\section{RESULTS}

For our first step, the specific heat of $\mathrm{CsNiF}_{3}$ was studied from $2 \mathrm{~K}$ to $5 \mathrm{~K}$ in zero magnetic field. These results were compared with the data reported by other workers, ${ }^{20}$ and the comparison is presented in Fig. 1. Both sets of data are characterized by a $\lambda$-like anomaly at about $2.7 \mathrm{~K}$, indicating the presence of long-range ordering. However, the larger peak observed in our experiment suggests that our single crystal specimen was of higher quality than the one used in the other work. ${ }^{20}$ The excellent agreement of the critical temperatures confirms that the silver epoxy did not deteriorate the bulk properties of our sample. The difference between both data sets can be described by the equation

$$
C(T)=a T+b T^{3}
$$

when $a=0.9 \pm 0.3 \mathrm{~J} / \mathrm{K}^{2}$ and $b=0.88 \pm 0.03 \mathrm{~J} / \mathrm{K}^{4}$. This difference is attributed to the specific heat of the addenda created predominantly by the vespel holder and the sapphire substrate. In addition, since the possible amount of paramagnetic impurities in the silver epoxy is at most $0.001 \%,{ }^{21}$ the specific heat of the addenda should have a negligible magnetic field dependence. The fact that the lattice contribution of $\mathrm{CsNiF}_{3}$ was not subtracted from the total heat capacity does not compromise the evaluation of the magnetic field dependence of the excess specific heat, the quantity of primary interest in this study.

The magnetic field dependence of the specific heat was studied at $2.4 \mathrm{~K}, 2.9 \mathrm{~K}$, and $4.2 \mathrm{~K}$, in fields up to $9 \mathrm{~T}$. As mentioned previously, the weak magnetoresistance of the heater is simple to accommodate and has no direct influence in a thermal relaxation study. Similarly, the weak magnetoresistance of the thermometer is negligible 
since it does not influence the thermal relaxation time of the calorimeter. On the other hand, the data were corrected using the known magnetoresistance of the Cernox thermometer. ${ }^{22}$ As expected, the correction was most significant at $T=2.4 \mathrm{~K}$ in magnetic fields higher than $6 \mathrm{~T}$, where it represented $3.5 \%$ of the measured heat capacity value. The resultant magnetic field dependence of the total specific heat is presented in Fig. 2. The $\lambda$-like anomaly observed at $2.4 \mathrm{~K}$ in a field of about $2 \mathrm{~T}$ corresponds to field-induced long-range ordering. The values of the critical temperature and field agree well with the magnetic phase diagram obtained from ultrasonic investigations of specimens from the same batch of crystals. ${ }^{16}$

\section{DISCUSSION}

For the investigation of the effect of the nonlinear excitations on the equilibrium thermodynamic properties, most of the attention has concentrated on the excess isothermal specific heat

$$
\Delta C(T, B)=C(T, B)-C(T, 0),
$$

studied as a function of magnetic field $B$ at a temperature $T$. The numerical predictions ${ }^{3}$ of the excess specific heat for $\mathrm{CsNiF}_{3}$ in a magnetic field oriented perpendicular to the easy plane were derived from a 1D model described by the $S=1$ Hamiltonian

$$
\mathcal{H}=-J \sum_{i} S_{i} \cdot S_{i+1}+D \sum_{i}\left({S_{i}}^{z}\right)^{2}+g \mu_{B} B \sum_{i} S_{i}{ }^{z}
$$

using the values $J / k_{B}=23.6 \mathrm{~K}, D / k_{B}=8.25 \mathrm{~K}$, and $g=2.13$. The numerical simulations were performed for temperatures $2.4 \mathrm{~K}$ and $4.2 \mathrm{~K}$, the former being below the critical temperature of the real material. The results of the numerical simulations suggest that the predicted double peak structure in the excess specific heat becomes more pronounced at lower temperatures. Consequently, in the corresponding experimental study, the influence of interchain correlations should be considered in the critical region.

The ratio of intrachain and interchain exchange coupling constants $\left(J / J^{\prime} \approx 500\right)$ makes $\mathrm{CsNiF}_{3}$ one of the best representatives of a quantum spin chain. ${ }^{23}$ Indeed, the long-range ordering observed at $2.66 \pm 0.01 \mathrm{~K}$ in this study is manifested as a small spike located on the broad maximum appearing due to the short-range correlations. In addition, the amount of entropy removed by the spike itself $(\approx 0.05 \mathrm{~J} /(\mathrm{K} \mathrm{mol}))$ is by far less than $1 \%$ of the total amount of entropy for the $S=1$ system, namely $9.13 \mathrm{~J} /(\mathrm{K} \mathrm{mol})$. In such a situation, it appears reasonable to approximate the contribution of the interchain coupling to the total specific heat by fitting the temperature dependence of the specific heat using data below and above the spike, i.e. outside the critical region. The result of this approximation is shown as the solid line in Fig. 1, and the enhancement of the specific heat in the critical region due to the interchain correlations is apparent (see inset in Fig. 1). Such an enhancement generates a deviation from the corresponding theoretical predictions, ${ }^{3}$ which were made for a purely $1 \mathrm{D}$ system. Consequently, when comparing the experimental excess specific heat data obtained at $2.4 \mathrm{~K}$ with the theory, the specific heat value calculated from the aforementioned fit at $2.4 \mathrm{~K}$ was taken as the reference value $C(T, 0)$ in Eq. (2). As a result, if the experimental specific heat data are represented by $C(T, B)$ in Eq. (2), then the quantity $\Delta C$ should tend to a nonzero value in the zero field limit. In other words, in the critical region, the interchain coupling is responsible for the finite value of the excess specific heat. The comparisons of the experimental excess specific heat data with the numerical predictions are presented in Fig. 3. As expected at $2.4 \mathrm{~K}$, the agreement between the theory and experiment is greatly improved in magnetic fields greater than $\approx 3 \mathrm{~T}$, which is high enough to overcome the influence of the long-range correlations and decompose the system into independent chains. This difference between the experimental data and theoretical predictions persists, but is smaller, at $2.9 \mathrm{~K}$, and it disappears at $4.2 \mathrm{~K}$. It should be noted that critical magnetic field observed at $2.4 \mathrm{~K}$ is consistent with the phase diagram obtained from the ultrasonic study which used specimens from the same batch of crystals. ${ }^{16}$ Furthermore, the experimental data reproduce the predicted positions of the minimum values of $\Delta C$, as well as the shift of the position of the second maximum towards lower fields with decreasing temperature. Although some quantitative differences persist, it should be stressed that the overall agreement between the numerical predictions and experimental data is better than that achieved when the magnetic field is oriented in the easy plane. ${ }^{3}$

Several potential reasons might explain the observed differences. For example, quantitative comparisons between the experimental data and the theoretical predictions should take demagnetizing effects into account. The correction of the specific heat due to demagnetizing effects can be calculated as follows. ${ }^{24}$ The internal magnetic field is written as

$$
B_{i}=B_{e}-N M
$$

where $B_{i}$ and $B_{e}$ stand for the internal and external fields, while $M$ and $N$ denote the magnetization and demagnetizing factor. The specific heat of the sample may then be written as

$$
C_{B_{i}}\left(T, B_{i}\right)=C_{B_{e}}\left(T, B_{i}\right)+\frac{\left[N T(\partial M / \partial T)_{B_{e}}^{2}\right]}{\left[1-N\left(\partial M / \partial B_{e}\right)_{T}\right]} .
$$

The needle shape of the sample, which was oriented with its long axis parallel with the applied external magnetic field, should lead to a small value of the demagnetizing factor. Indeed, for the dimensions of our single crystal 
specimen, the average demagnetizing factor is approximately 0.04 in SI units. In order to evaluate $\left(\partial M / \partial B_{e}\right)_{T}$, the field dependence of magnetization was investigated up to $7 \mathrm{~T}$ at $2.4 \mathrm{~K}, 2.9 \mathrm{~K}$, and $4.2 \mathrm{~K}$. For clarity, only the results obtained at $2.4 \mathrm{~K}$ and $4.2 \mathrm{~K}$ are presented in Fig. 4. For all temperatures, the magnetization is characterized by an increase up to the critical field, followed by the tendency to saturate at higher fields. It should also be noted that, for the magnetization data corrected for demagnetizing effects, some deviation from the numerical predictions appears. The values of the term $(\partial M / \partial T)_{B_{e}}$ were evaluated with the help of the additional field dependences of the magnetization studied at temperatures about $2 \%$ higher and lower than the aforementioned ones. Then $(\partial M / \partial T)_{B_{e}}$ for $2.4 \mathrm{~K}, 2.9 \mathrm{~K}$, and $4.2 \mathrm{~K}$ were approximated by the corresponding differences of the magnetization at the neighboring temperatures. The results of this analysis are presented in Fig. 5. The remarkable feature of the $(\partial M / \partial T)_{B_{e}}$ quantity is its nontrivial field dependence in the region of low fields, where it is most pronounced at $2.4 \mathrm{~K}$ and also observed at $2.9 \mathrm{~K}$, but is completely absent at $4.2 \mathrm{~K}$. Taking into account that both $2.4 \mathrm{~K}$ and $2.9 \mathrm{~K}$ belong to the critical region of temperatures for $\mathrm{CsNiF}_{3}$, the observed behavior can be attributed to the effect of interchain coupling. It should be noted that, for $2.4 \mathrm{~K}$ and $2.9 \mathrm{~K}$, the value of the field, where the anomalous behavior of $(\partial M / \partial T)_{B_{e}}$ disappears $(\approx 3 \mathrm{~T})$, agrees very well with that for which $\Delta C$ starts to follow the theoretical prediction for a pure 1D system. Such agreement supports the suggestion that $\approx 3 \mathrm{~T}$ is sufficient to suppress the $3 \mathrm{D}$ effects near $T_{N}$. After evaluating $(\partial M / \partial B)_{T}$ and $(\partial M / \partial T)_{B_{e}}$, the correction to the specific heat due to the demagnetizing effects was calculated. However, it turns out that the correction does not exceed $0.1 \%$ of the uncorrected specific heat value, thus demagnetizing effects can not be responsible for the observed differences. This fact contrasts with the situation when magnetic field is oriented in the easy plane, where such a correction was proven to be significant. ${ }^{25}$

Naturally, tuning the parameters $J, D$, and $g$ might improve the agreement between the numerical predictions and the experimental results. Consequently, the susceptibility, ${ }^{14}$ magnetization, ${ }^{15}$ and present specific heat data were reanalyzed using the transfer matrix renormalization group technique. Since no evidence for the change of exchange coupling constant was found in the previous analysis, ${ }^{3}$ the value $J / k_{B}=23.6 \mathrm{~K}$ was adopted in the recalculation. In the new set of parameters, the 'standard' value of $D / k_{B}=9 \mathrm{~K}$ was chosen, whereas $g=2.19$ was obtained from fitting the susceptibility $^{14}$ and magnetization ${ }^{15}$ with the new $D$ value. It should be noted that such a choice of new $D$ and $g$ parameters does not significantly change the magnitude of the critical field, $B_{c}=D / g \mu_{B} \approx 6 \mathrm{~T}$ as suggested by the specific heat data. However, as can be seen in Fig. 3, the new predictions are shifted towards larger fields and the values of $\Delta C$ are increased, thereby making the agreement with the experimental data worse than for the original set of parameters. Further increasing of the $D$ value leads to unsatisfactory fitting of the susceptibility and magnetization. Consequently, tuning the parameters in the framework of a pure $1 \mathrm{D}$ model does not suppress the differences between the numerical prediction and the experimentally measured excess specific heat.

Finally, the possible effect of the tilting of the c-axis with respect to the direction of the magnetic field may be considered. It is not straightforward to quantitatively evaluate this effect, but it can be expected to influence the experimental results. For example, the susceptibility below $8 \mathrm{~K}$ is about five times higher for magnetic field oriented in the easy plane than that for the perpendicular orientation. ${ }^{3}$ Although the magnitudes of $\Delta C$ are comparable for the different field orientations, the corresponding field dependences are completely different. Thus, tilting may nontrivially contribute to the differences between the numerical predictions and experimental specific heat data.

\section{CONCLUSIONS}

The experimental study of the magnetic field dependence of excess specific heat performed at $2.4 \mathrm{~K}, 2.9 \mathrm{~K}$, and $4.2 \mathrm{~K}$ has confirmed the theoretically predicted double peak structure reflecting the nonlinear behavior of the system. The excess specific heat data reproduce the predicted shift of the second maximum towards lower fields with decreasing temperature. It was found that, similarly as for the orientation of the magnetic field in the easy plane, it is not possible to fit all available thermodynamic data using a pure 1D model with a single set of parameters. Although tilting of the sample may be an alternative explanation of the persisting deviations, demagnetizing effects are determined to be negligible. This conclusion contrasts with the one made in Ref. 6, where the magnetic field was oriented in the easy plane and where the correction for the shape dependence had a pronounced effect. However, even with the incorporation of the demagnetization correction, the agreement between the excess specific heat data ${ }^{6}$ and the corresponding exact numerical predictions ${ }^{3}$ is worse than in our case. In Ref. 6 , the estimation of $(\partial M / \partial T)_{B_{e}}$ from the field dependence of the magnetization ${ }^{26}$ might not be sufficiently accurate due to the restricted amount of data that is available. Indeed, in situations where the demagnetizing effects are pronounced, more detailed mappings of the field and temperature dependences of the magnetization were performed. ${ }^{27}$ Naturally, a question may arise about the influence of demagnetizing effects in the specific heat studies of other soliton - bearing systems. A considerable amount of theoretical effort ${ }^{28-30}$ has been developed to understand the differences and to improve the agreement with the specific heat data. ${ }^{11,31}$ However, to the best of our knowledge, the data themselves were not corrected 
for demagnetizing effects. Consequently, a future experiment might focus on clarifying the role of the demagnetizing effects in $\mathrm{CsNiF}_{3}$ as a soliton - bearing system. Furthermore, since the predicted double peak structure seems to be a generic feature of the nonlinear behavior in classical and quantum spin chains, future experimental effort may focus on measuring $\Delta C$ in other related materials.

\section{ACKNOWLEDGMENTS}

We would like to thank N. Papanicolaou for enlightening discussions and for providing us with the results of numerical calculations published in Ref. 3. Early contributions from B. C. Watson during the design and construction of the probe, and B. Andraka during the initial phases of the work, are gratefully acknowledged. This work was supported, in part, by the National Science Foundation (INT-0089140, DMR-0113714, DGE-0209410, and DMR-0305371) and the Slovak Grant Agency (VEGA 1/0430/03).

${ }^{1}$ H. J. Mikeska, J. Phys. C 11, L29 (1978).

${ }^{2}$ J. M. Wesselinowa and V. P. Ivanova, Phys. Rev. B 54, 9846 (1996).

${ }^{3}$ J. Karadamoglou, N. Papanicolaou, X. Wang, and X. Zotos, Phys. Rev. B 63, 224406 (2001) and references therein.

${ }^{4}$ M. Steiner, K. Kakurai, and J. K. Kjems, Z. Phys. B 53, 117 (1983).

${ }^{5}$ T. Goto and Y. Yameguchi, J. Phys. Soc. Jpn. 50, 2133 (1981).

${ }^{6}$ A. P. Ramirez and W. P. Wolf, Phys. Rev. Lett. 49, 227 (1982).

7 D. H. Reich, L. P. Lévy, and T. Giamarchi, Phys. Rev. Lett. 67, 2207 (1991).

${ }^{8}$ G. Reiter, Phys. Rev. Lett. 46, 202 (1981).

${ }^{9}$ O. G. Mouritsen, H. Jensen, and H. C. Fogedby, Phys. Rev. B 30, 498 (1984).

${ }^{10}$ M. G. Pini and A. Rettori, Phys. Rev. B 29, 5246 (1984).

${ }^{11}$ F. Borsa, Phys. Rev. B 25, 3430 (1982).

12 J. Cibert and Y. M. d'Aubigné, Phys. Rev. Lett. 46, 1428 (1981).

${ }^{13}$ P. S. Riseborough and P. Kumar, J. Phys. C 21, L185 (1988).

${ }^{14}$ C. Dupas and J. P. Renard, J. Phys. C 10, 5057 (1977).

15 T. Delica, W. J. M. de Jonge, K. Kopinga, H. Leschke, and H. J. Mikeska, Phys. Rev. B 44, 11773 (1991).

${ }^{16}$ B. Lussier and M. Poirier, Phys. Rev. B 48, 6199 (1993).

${ }^{17}$ C. Pich and F. Schwabl, J. Appl. Phys. 81, 4623 (1997).

18 N. Papanicolaou and P. Spathis, Z. Phys. B 65, 329 (1987).

${ }^{19}$ N. Papanicolaou and P. Spathis, J. Phys. C. 20, L783 (1987).
${ }^{20}$ J. V. Lebesque, J. Snel, and J. J. Smit, Solid State Comm. 13, 371 (1973).

${ }^{21}$ Epotek, private communication.

${ }^{22}$ B. L. Brandt, D. W. Liu, and L. G. Rubin, Rev. Sci. Instrum. 70, 104 (1999).

${ }^{23}$ M. Baehr, M. Winkelmann, P. Vorderwisch, and M. Steiner, Physica B 234-236, 552 (1997).

${ }^{24}$ P. M. Levy and D. P. Landau, J. Appl. Phys. 39, 1128 (1968).

${ }^{25}$ A. P. Ramirez and W. P. Wolf, Phys. Rev. B 32, 1639 (1985).

${ }^{26}$ Ch. Rosinski and B. Elschner, J. Magn. Magn. Mater. 4, 193 (1977).

27 D. P. Landau, B. E. Keen, B. Schneider, and W. P. Wolf, Phys. Rev. B 3, 2310 (1971).

28 A. M. C. Tinus, W. J. M de Jonge, and K. Kopinga, Phys. Rev. B 32, 3154 (1985).

${ }^{29}$ H. J. Jensen, O. G. Mouritsen, H. C. Fogedby, P. Hedegard, and A. Svane, Phys. Rev. B 32, 3240 (1985), and references therein.

${ }^{30}$ A. M. C. Tinus, W. J. M. de Jonge, and K. Kopinga, J. Magn. Magn. Mater. 54-47, 824 (1986).

${ }^{31}$ K. Kopinga, A. M. C. Tinus, and W. J. M. de Jonge, Phys. Rev. B 29, 2868 (1984).

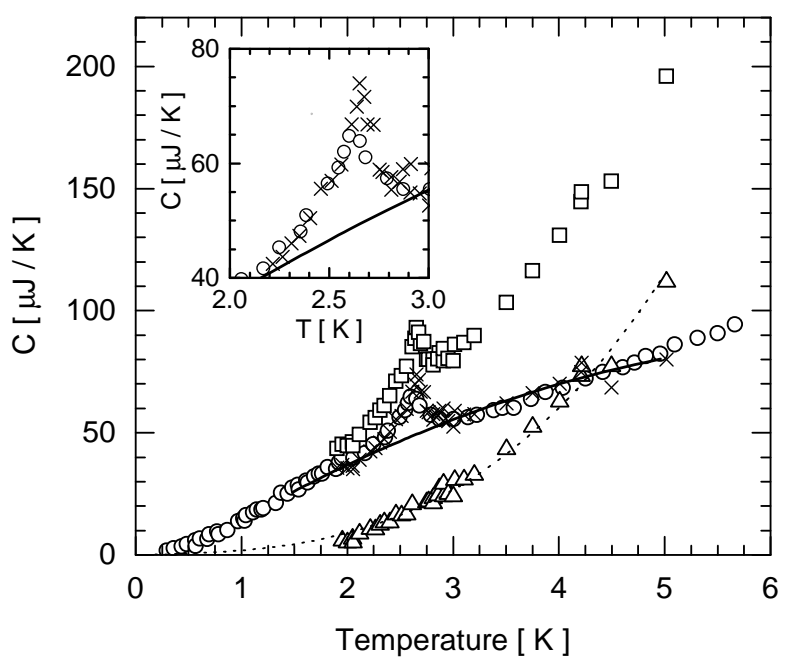

FIG. 1. The temperature dependence of total heat capacity of $\mathrm{CsNiF}_{3}$ together with the addenda (squares) is compared to the data of magnetic heat capacity reported in Ref. 20 (circles), after renormalization to the mass of our sample. The estimated contribution of the addenda for the present study is shown by the triangles. The heat capacity of our sample, i.e. the difference between the squares and triangles, is represented by the crosses. A least square fit of the lattice contribution and the addenda is denoted by the dotted line, see Eq. (1). The solid line is obtained by fitting the data outside the critical region, see the text for a more detailed discussion. The inset provides an expanded view near the critical region. 


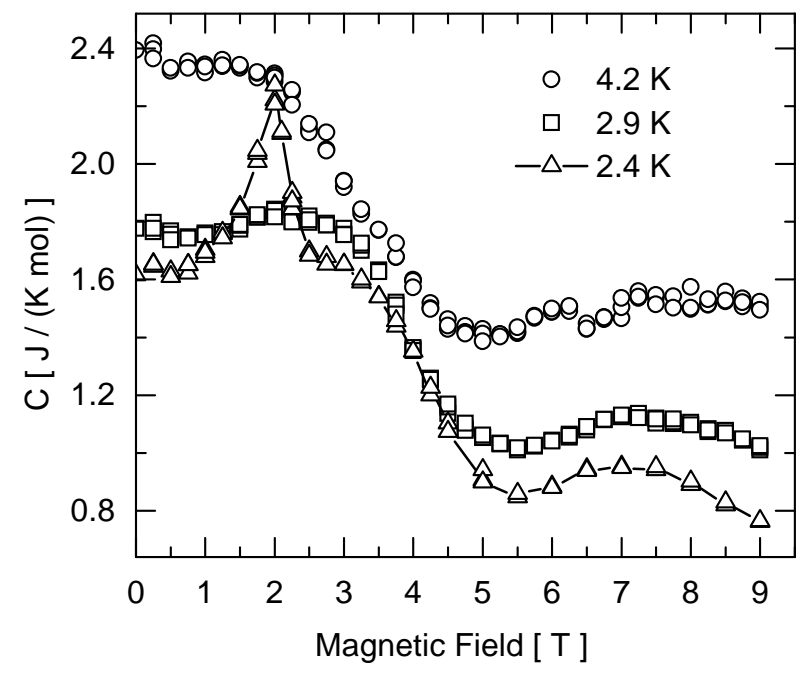

FIG. 2. The magnetic field dependence of the total specific heat of $\mathrm{CsNiF}_{3}$ is shown at $2.4 \mathrm{~K}$ (triangles with lines as guides for the eyes), $2.9 \mathrm{~K}$ (squares) and $4.2 \mathrm{~K}$ (circles).

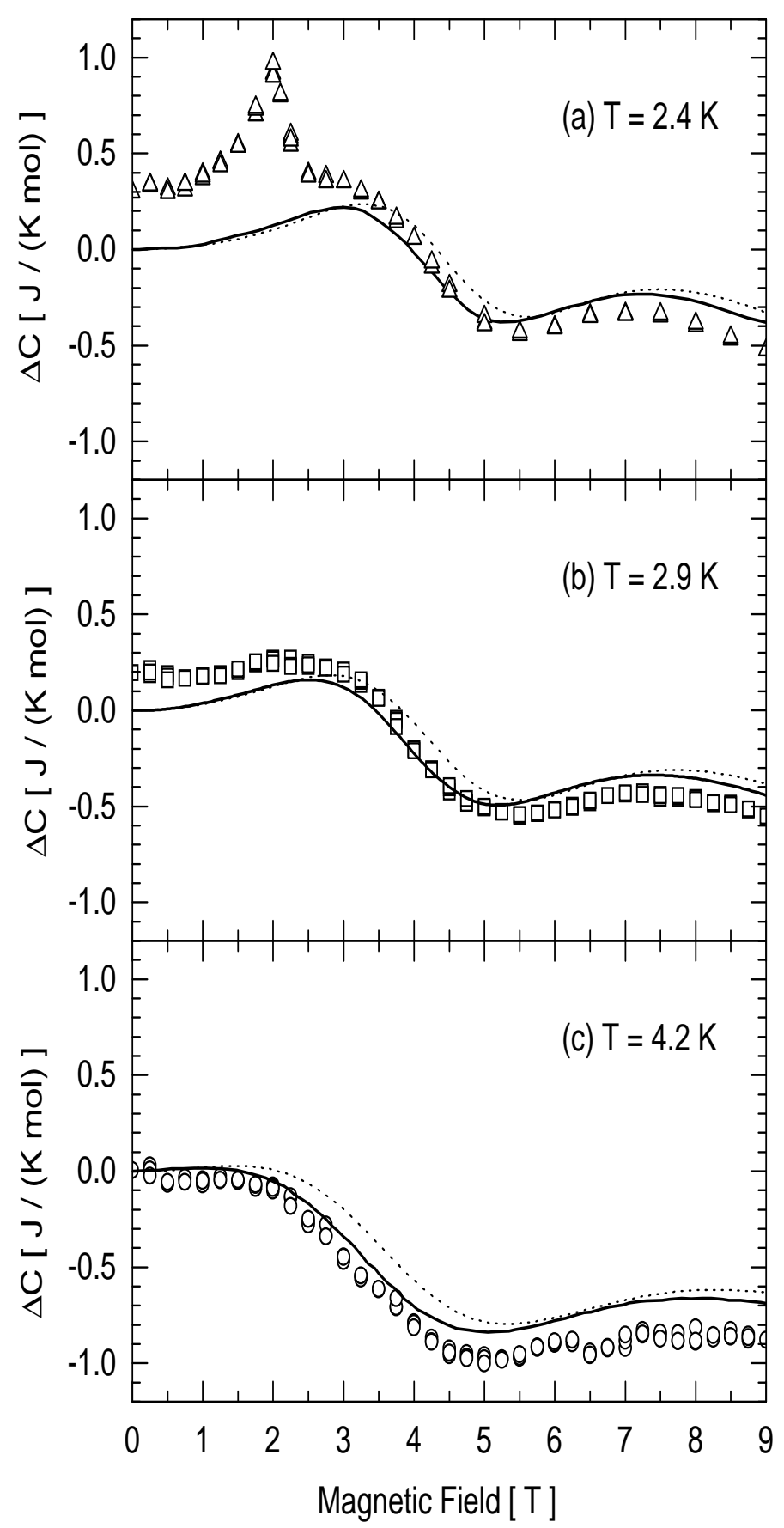

FIG. 3. The magnetic field dependence of $\Delta C$, Eq. (2), at (a) $T=2.4 \mathrm{~K}$, (b) $T=2.9 \mathrm{~K}$, and (c) $T=4.2 \mathrm{~K}$, are compared with theoretical expectations. The solid lines represent the numerical predictions when $J / k_{B}=23.6 \mathrm{~K}$, $D / k_{B}=8.25 \mathrm{~K}$, and $g=2.13$, while the dotted lines are for $J / k_{B}=23.6 \mathrm{~K}, D / k_{B}=9 \mathrm{~K}$, and $g=2.19$. 


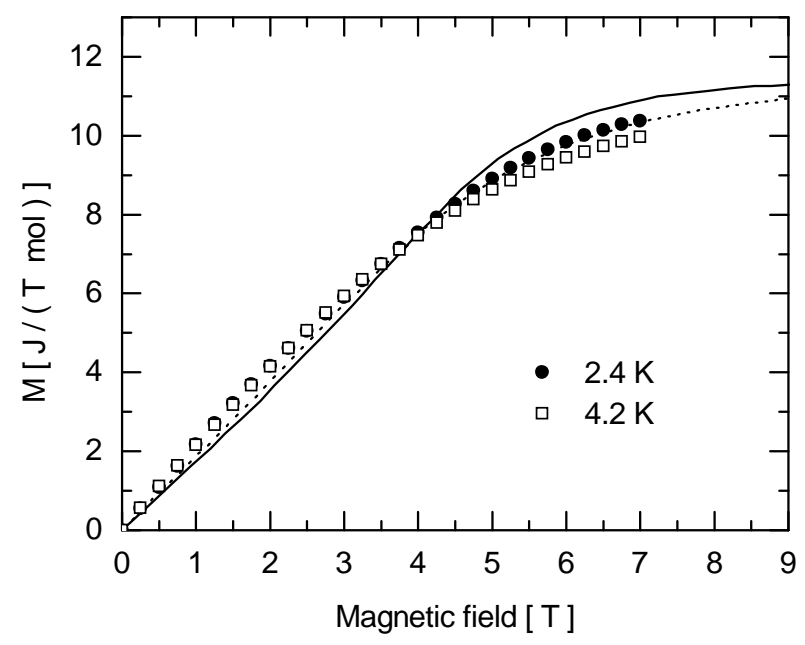

FIG. 4. Magnetic field dependence of magnetization of $\mathrm{CsNiF}_{3}$ studied at $2.4 \mathrm{~K}$ (squares) and $4.2 \mathrm{~K}$ (circles). The solid and dotted lines represent the numerical predictions at $2.4 \mathrm{~K}$ and $4.2 \mathrm{~K}$, respectively, when $J / k_{B}=23.6 \mathrm{~K}$, $D / k_{B}=8.25 \mathrm{~K}$, and $g=2.13$.

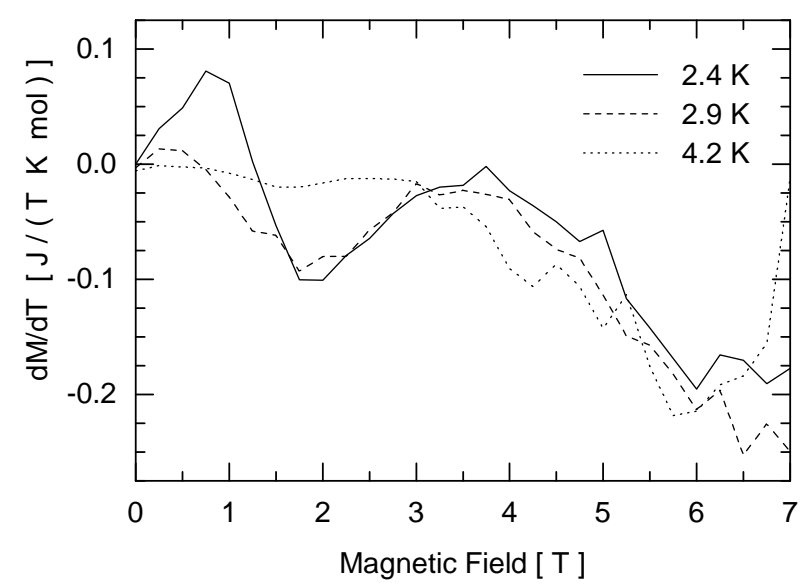

FIG. 5. Magnetic field dependence of $(\partial M / \partial T)_{B_{e}}$ calculated at $2.4 \mathrm{~K}$ (solid line), $2.9 \mathrm{~K}$ (dashed line), and $4.2 \mathrm{~K}$ (dotted line). 